\title{
ATIVIDADE DE APRENDIZAGEM BASEADA EM PROJETO EM ENGENHARIA CIVIL: PROPOSTA DE ANÁLISE EXPERIMENTAL DO MÓDULO DE ELASTICIDADE E DIMENSIONAMENTO DE VIGA
}

DOI: 10.37702/2175-957X.COBENGE.2021.3549

ELIEDSON RAFAEL DE CARVALHO - eliedson.carvalho@ifal.edu.br Instituto Federal de Alagoas Av. Alagoas - Palmeira de Fora $s / n$ 57608-180 - Palmeira dos Índios - AL

JULIANA LIRA BRITO - julianaifal@hotmail.com Instituto Federal de Alagoas RUA COSTA GAMA 234 57304-250 - ARAPIRACA - AL

Dackyson Kelwyn de Souza Lopes Holanda - dackysonkholanda@gmail.com Instituto Federal de Alagoas

Rua Vereador Eustáquio Soares da Silva 205

57750-000 - Quebrangulo - AL

João Vitor Paixão Silva - jv.paixaosilva@gmail.com Instituto Federal de Alagoas

Rua Ângelo Tavares 160

57305-390 - Arapiraca - AL

Eliana Queiroz de Castro - eliana5.0c@gmail.com

Instituto Federal de Alagoas

Sítio Cascavel S/N

57625-000 - Estrela de Alagoas - AL

Resumo: Este artigo tem como objetivo apresentar os resultados obtidos na atividade aplicada na turma de Mecânica dos Sólidos III do curso de Engenharia Civil do Instituto Federal de Alagoas - IFAL, utilizando conceitos da Aprendizagem Baseada em Projeto - ABP como alternativa metodológica durante o ensino remoto emergencial. De maneira geral, os procedimentos metodológicos da Atividade de $A B P$ podem ser divididos em quatro etapas: delimitação da situação problema, 
protótipo didático de experimento do módulo de elasticidade, projeto da viga e análise de tensões. Inicialmente, os estudantes receberam a proposta da atividade que consiste no dimensionamento da viga de suporte para uma placa a partir do material de um objeto escolhido que neste caso foi uma régua polimérica comum. Além disso, os estudantes adotaram as dimensões e material da placa. A partir da régua, os estudantes construíram o protótipo didático para realização de experimento para determinação do módulo de elasticidade do material, utilizando os conceitos de mecânica dos sólidos trabalhados durante a disciplina. Com base no valor médio do módulo de elasticidade do material e os dados adotados, foi possível dimensionar a viga de suporte placa e, em seguida, elaborar o projeto. $A$ partir dos parâmetros de projeto, foi realizada a análise de tensões na viga. De modo geral, os resultados da Atividade, enquanto proposta metodológica, podem ser considerados satisfatórios, pois os estudantes conseguiram resolver a situação problema proposta, elaborar o projeto e analisar a viga, relacionando teoria e prática.

Palavras-chave: Aprendizagem baseada em projeto. Mecânica dos sólidos. Viga. 


\section{ATIVIDADE DE APRENDIZAGEM BASEADA EM PROJETO EM ENGENHARIA CIVIL: PROPOSTA DE ANÁLISE EXPERIMENTAL DO MÓDULO DE ELASTICIDADE E DIMENSIONAMENTO DE VIGA}

\section{INTRODUÇÃO}

A indústria da construção civil tem adotado a utilização de softwares com o objetivo de estender as possibilidades de análise durante os processos de simulação e cálculo estrutural, no entanto, é necessário que o calculista entenda o processo e o método para que não ocorra nenhum contratempo, como superdimensionamento ou subdimensionamento, e, dessa forma, caracterize qualquer tipo de prejuízo à obra (KRÜGER; DA SILVA, 2019).

Segundo Chistoforo e Ribeiro Filho (2013), para o dimensionamento de uma estrutura, o módulo de elasticidade do material é uma das mais importantes propriedades a serem analisadas durante o processo. Visto que os projetos estruturais são realizados com o auxílio das propriedades mecânicas dos materiais, e a relação entre deformação plástica e tensão, adquirida através do módulo elasticidade, possui grande influência no desempenho estrutural.

De modo geral, durante a formação, o acadêmico em Engenharia Civil deve ser exposto a situações que o permitam desenvolver a capacidade entender todo 0 processo de dimensionamento e comportamento das estruturas. Atrelado a isso, Ibáñez e Delgado-Kloos (2018) revelam que as áreas das Ciências, Tecnologia, Engenharia e Matemática (STEM) demandam habilidades específicas por parte dos estudantes para o pleno processo de ensino-aprendizagem, como: capacidade espacial, habilidades práticas, compreensão conceitual e investigação científica.

Estudantes que optam por cursos nessas áreas acabam enfrentando dificuldades para compreender determinados conteúdos associadas ao fato de não conseguirem desenvolver de forma efetiva as habilidades supracitadas. Além disso, é possível acrescentar no processo de ensino-aprendizagem de engenharia, as dificuldades apresentadas pelo professor ao tentar contextualizar os conteúdos de forma prática, próxima a realidade dos estudantes (FEITOSA, 2020).

Para Daineko et al. (2017), a inserção de novas tecnologias de informação e comunicação, combinadas com metodologias ativas utilizadas no processo de ensinoaprendizagem, tem contribuído para o aumento significativo da experiência de ensino e melhorado a qualidade da educação.

Dentre as metodologias ativas, pode-se destacar a Aprendizagem Baseada em Projetos - ABP (Project Based Learning - PBL) a qual, permite que diferentes grupos de estudantes confrontem questões e problemas do mundo reais significativos para eles, determinando assim a maneira mais prática de abordá-los e estabeleçam ação cooperativa em busca de soluções. Cipolla (2016) salienta que este método de aprendizagem consegue acomodar mudanças e atualizações tecnológicas que estão em andamento, servindo de interface para que os estudantes desenvolvam as habilidades nessas inovações que se dão constantemente.

Neste contexto, a ABP surge como forte aliada no processo de ensino das disciplinas do curso de engenharia civil, pois permite ao acadêmico agregar conhecimento e desenvolver habilidades através da busca por solução de problemas baseados em situações que os aproximam da prática profissional.

Por fim, este artigo tem como objetivo apresentar os resultados obtidos na Atividade aplicada na disciplina de Mecânica dos Sólidos III utilizando conceitos da 
Aprendizagem Baseada em Projeto como alternativa metodológica durante o ensino remoto emergencial.

\section{MATERIAIS E MÉTODOS}

Visando trazer os resultados adquiridos na Atividade utilizando ABP realizada na disciplina do Mecânica dos Sólidos III do Instituto Federal de Alagoas - IFAL Campus Palmeira dos Índios, este trabalho apresenta metodologia com enfoque quantitativo e descritivo, possuindo como instrumento a realização de experimentos.

De maneira geral, os procedimentos metodológicos da Atividade de ABP podem ser divididos em quatro etapas: delimitação da situação problema, protótipo didático de experimento do módulo de elasticidade, projeto da viga e análise de tensões.

\subsection{Delimitação da situação problema}

A situação problema principal gira em torno da elaboração do projeto para viga para sustentação de placa. A princípio, os estudantes selecionaram o material da viga e, em seguida, construíram o protótipo didático de experimento para determinação do módulo de elasticidade do material escolhido.

As dimensões e o material da placa foram adotados pelos estudantes, uma vez que essas informações seriam necessárias para o levantamento dos carregamentos e condições de contorno do projeto.

Com base na teoria discutida durante a disciplina e das informações coletadas no protótipo didático de experimento, os estudantes dimensionaram a viga a partir da flecha máxima permitida recomendada pela norma brasileira NBR 15.575 (ABNT, 2013) que prevê o deslocamento-limite para vigas estruturais de $L / 250$, no qual $L$ é 0 comprimento da viga. Com a viga dimensionada, foi possível prosseguir com a realização da análise de tensões e a elaboração do projeto da viga.

\subsection{Protótipo didático de experimento}

Para o material da viga, foi escolhido um material polimérico proveniente de uma régua comum com $50 \mathrm{~cm}$ de comprimento. Os materiais e equipamentos utilizados no procedimento experimental para obtenção do módulo de elasticidade do material escolhido foram: régua polimérica, com comprimento de $50 \mathrm{~cm} ; 2$ parafusos; chave de fenda; suporte metálico; pesos; sacola plástica; smartphone; apoio de madeira.

Inicialmente, foi preparado o protótipo estrutural utilizando a régua de maneira similar à viga com extremidade engasta e outra livre. Para isso, colocou-se os parafusos ligando a extremidade engastada da régua ao apoio de madeira. A Figura 1 mostra a vista lateral do protótipo.

Figura1 - Vista lateral do protótipo

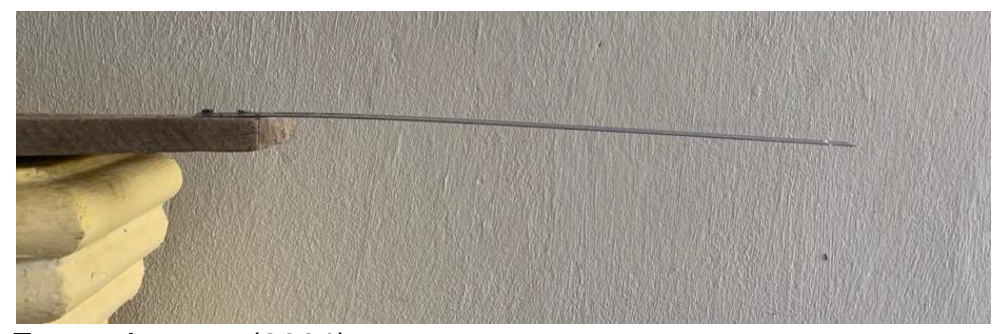

Fonte: Autores (2021) 
Para análise da variação da inclinação da régua em relação ao plano inicial ao ser carregada com os pesos, utilizou-se o software Phyphox através do equipamento smartphone.

Após montagem e calibração dos equipamentos, adicionou-se progressivamente os pesos na sacola plástica até que houvesse uma considerável inclinação da régua em relação ao estado inicial. Em seguida, coletou-se os dados obtidos pelo software durante o experimento e realizou-se o estudo do comportamento elástico do material, com posterior obtenção do módulo de elasticidade.

Vale ressaltar que o procedimento foi construído em ambiente com baixo nível de controle e todo experimento foi moldado para fins estritamente didático, visto a impossibilidade de acesso aos laboratórios.

Assim, como a proposta da atividade é estritamente didática, foram utilizadas as equações apresentadas por Hibbeler (2010) para determinação do módulo de elasticidade, mesmo entendendo que o comportamento da régua não atende plenamente a todas condições para a aplicação das equações. Neste caso, a Equação 1 representa a inclinação na extremidade livre para a situação adotada (viga engastada).

$$
\theta=\frac{P L^{2}}{2 E I}
$$

Na qual, $\boldsymbol{\theta}$ é a inclinação em graus, $P=$ peso em $\mathrm{N}, I=$ momento de inércia da seção em $m^{4}$, $L$ é o comprimento em metro e $E$ = módulo de elasticidade em $P a$.

Assim, os dados referentes a inclinação para cada peso foram anotados e o módulo de elasticidade foi determinado por meio da Equação 1. A partir dos resultados, determinou-se o valor médio para o módulo de elasticidade.

\subsection{Projeto da viga}

Para obtenção dos parâmetros estruturais relacionados à viga do modelo teórico abordado no projeto, foi considerado o conjunto de elementos responsáveis pelos carregamentos na estrutura. A princípio, a viga foi modelada com seção transversal circular com uma extremidade engastada. Complementar à estrutura, foi adotado o modelo de placa circular à base da madeira maçaranduba, apoiada à viga através de dois cabos de aço. Desse modo, foram atribuídos os valores de entrada da Tabela 1 para o projeto.

Tabela 1 - Valores de entrada do modelo teórico

\begin{tabular}{ccc}
\hline \multicolumn{2}{c}{ Placa Circular } & Viga \\
\hline Diâmetro $(\mathrm{m})$ & Profundidade $(\mathrm{m})$ & Comprimento $(\mathrm{m})$ \\
\hline 0,50 & 0,10 & $0,80 \mathrm{~m}$ \\
\hline Fonte: Autores $(2021)$ &
\end{tabular}

Para o melhor entendimento, a Figura 2 traz a representação esquemática do modelo proposto. 
Figura 2 - Vista de perfil do modelo teórico

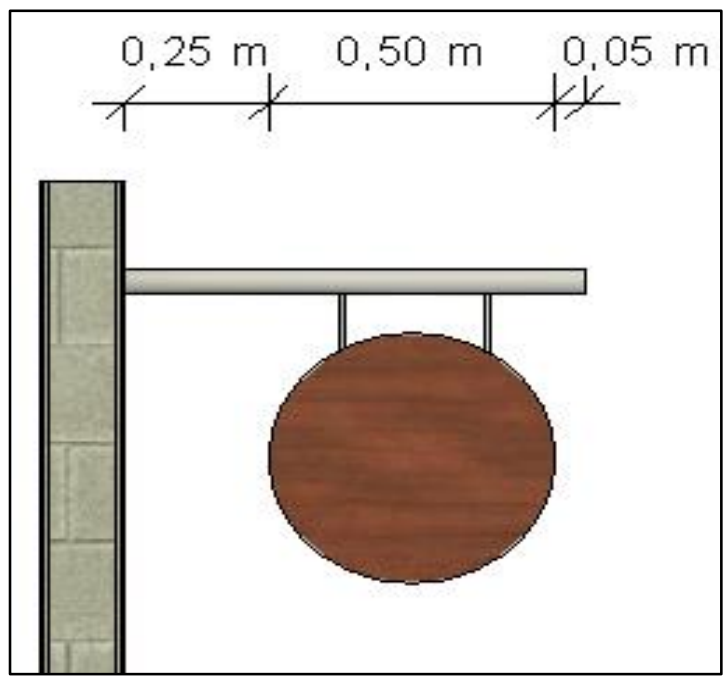

Fonte: Autores (2021)

Após determinação da conjuntura estrutural, realizou-se os cálculos necessários para obtenção dos parâmetros pendentes da placa circular, compostos pelo volume, massa e peso realizada em cada cabo de aço. O carregamento da viga é composto por duas cargas concentradas $T$ calculadas a partir do peso próprio da placa (Pp). Por se encontrarem de forma simétrica em relação ao centro de carga da placa, a carga de T por ser calculada pela Equação 2.

$$
T=\frac{P_{p}}{2}
$$

A partir das dimensões bases e dos carregamentos, o modelo teórico ficou com a configuração da Figura 3.

Figura 3 - Distribuição de esforços e seções de corte analisadas na estrutura

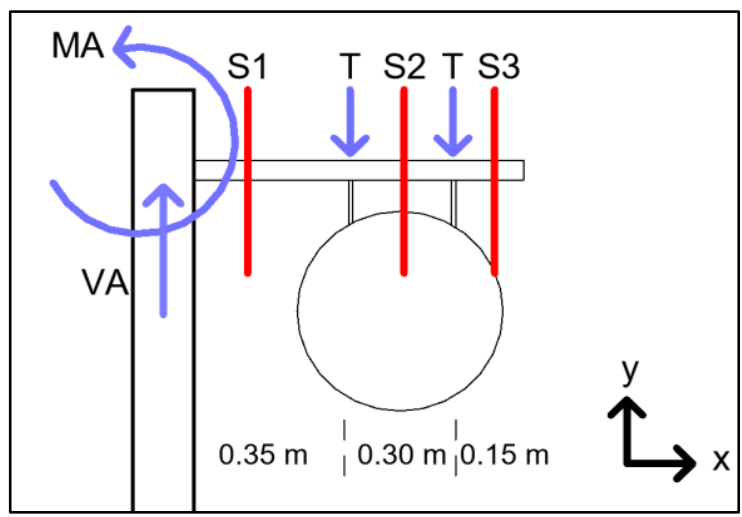

Fonte: Autores (2021)

Com base no modelo da Figura 3, foi possível determinar a deflexão máxima da estrutura (extremidade livre) em função do diâmetro da viga. A Equação 3 presente em Timoshenko e Gere (1983) foi utilizada para determinação da flecha na extremidade livre. 


$$
\delta=\frac{P a^{2}(3 L-a)}{6 E I}
$$

Na qual, $\delta$ é a deflexão na extremidade livre, $P$ é a carga concentrada, $L$ é comprimento da viga, a é a distância do ponto da carga concentrada até o gaste, $E$ é módulo de elasticidade e l é momento de inércia da seção.

A Figura 4 mostra o esquema utilizado para aplicação da Equação 3 no modelo teórico proposto.

Figura 4 - Partição da deformação total ao longo da estrutura

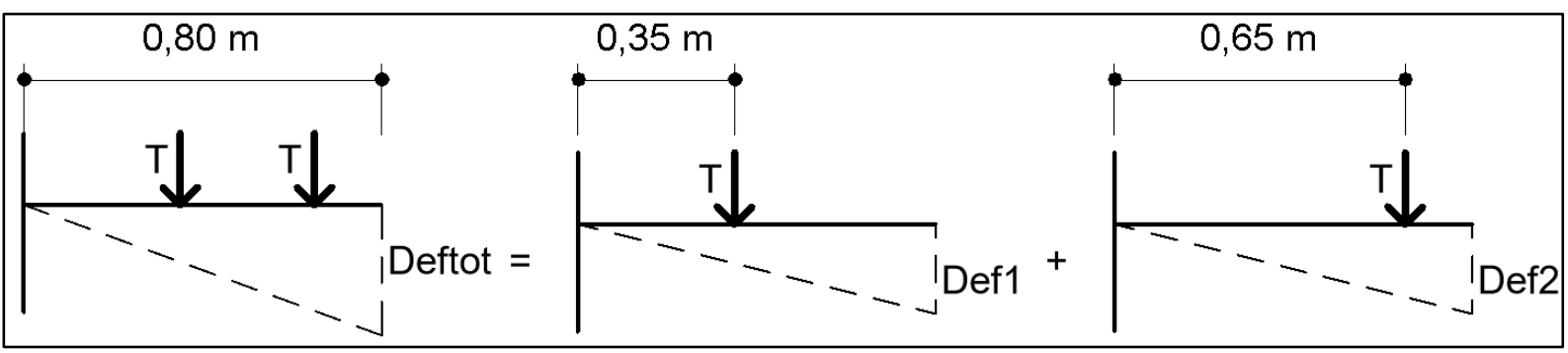

Fonte: Autores (2021)

Calculado o valor da deflexão total na extremidade livre, foi possível realizar a comparação com deslocamento-limite previsto pela NBR 15.575 (ABNT, 2013), L/250, para encontrar o diâmetro mínimo da viga. Desse modo, o dimensionamento da viga foi finalizado.

\subsection{Análise de Tensões}

Após o dimensionamento da viga, determinou-se as tensões máximas normais de tração e compressão e também a tensão máxima de cisalhamento. Para isso, foram utilizadas as Equações 4, 5 e 6 presentes em Beer et al. (2011).

$$
\begin{gathered}
\sigma_{\text {máx(tra) }}=\frac{M_{\text {máx }}}{I} \\
\sigma_{\text {máx(com) }}=-\sigma_{\text {máx }(t r a)} \\
\tau_{\text {máx }}=\frac{4 V_{\text {máx }}}{3 A}
\end{gathered}
$$

Na qual $\sigma_{m a ́ x(t r a)}$ é tensão normal máxima de tração, $\sigma_{\text {máx(com) }}$ é tensão normal máxima de compressão, Mmáx é o momento máximo, $c$ é a distância da linha neutra até o ponto de análise da tensão, $I$ é o momento de inércia, $A$ é a área da seção da viga, $\tau_{\text {máx }}$ é a tensão máxima de cisalhamento e $V_{\text {máx }}$ é o esforço cortante máximo.

Os dados referentes a análise de tensões foram computados para a construção de tabelas e gráficos.

\section{RESULTADOS E DISCUSSÃO}

A Tabela 2 apresenta os parâmetros da régua polimérica utilizada na análise experimental. Vale ressaltar que a régua foi utilizada no sentido de menor inércia como 
mostrado anteriormente (ver Figura 1) para facilitar a realização da análise experimental e coleta de dados.

Tabela 2 - Dimensões da régua polimérica utilizada para análise experimental

\begin{tabular}{cccc}
\hline $\mathbf{L}(\mathbf{m})$ & $\mathbf{h}(\mathbf{m})$ & $\mathbf{b}(\mathbf{m})$ & $\mathbf{I}\left(\boldsymbol{m}^{\mathbf{4}}\right)$ \\
\hline 0,46 & 0,003 & 0,03 & $\mathbf{6 , 7 5} \cdot \mathbf{1 0}^{-\mathbf{1 1}}$ \\
\hline
\end{tabular}

Fonte: Autores (2021)

A partir dos dados da Tabela 2, foi possível calcular o valor do momento de inércia, obtendo como resultado $6,75 \cdot 10^{-11} \mathrm{~m}^{4}$. O valor obtido foi adotado para aplicação no cálculo do módulo de elasticidade.

Tabela 3 - Dados obtidos durante análise experimental

\begin{tabular}{ccccc}
\hline Medição & Inclinação (rad) & Massa $(\mathbf{k g})$ & Peso $(\mathbf{N})$ & Peso acumulado $(\mathbf{N})$ \\
\hline $\mathbf{1}$ & 0,109 & 0,1940 & 1,901 & 1,901 \\
\hline $\mathbf{2}$ & 0,129 & 0,0095 & 0,093 & 1,994 \\
\hline $\mathbf{3}$ & 0,149 & 0,0095 & 0,093 & 2,087 \\
\hline $\mathbf{4}$ & 0,171 & 0,0095 & 0,093 & 2,181 \\
\hline $\mathbf{5}$ & 0,190 & 0,0095 & 0,093 & 2,274 \\
\hline $\mathbf{6}$ & 0,211 & 0,0095 & 0,093 & 2,367 \\
\hline
\end{tabular}

Fonte: Autores (2021)

A partir dos valores encontrados, durante a análise experimental, dos pesos acumulados e inclinações correspondentes (Tabela 3), juntamente com os valores fixos descritos na Tabela 2 para o momento de inércia e o comprimento da régua, foram determinados os valores de módulo de elasticidade para cada medição. A Tabela 4 traz os valores calculados para o módulo de elasticidade.

Tabela 4 - Módulo de elasticidade obtido referente à régua

\begin{tabular}{ccc}
\hline MEDIÇÃO & $\mathbf{E}(\mathbf{P a})$ & Eméd (Pa) \\
\hline $\mathbf{1}$ & $2,736 \cdot 10^{10}$ & \\
\hline $\mathbf{2}$ & $2,424 \cdot 10^{10}$ & \\
\hline $\mathbf{3}$ & $2,195 \cdot 10^{10}$ & \\
$\mathbf{4}$ & $1,998 \cdot 10^{10}$ & \\
\hline $\mathbf{5}$ & $1,873 \cdot 10^{10}$ & \\
$\mathbf{6}$ & $1,757 \cdot 10^{10}$ & \\
\hline
\end{tabular}

Fonte: Autores (2021)

Com isso, foi calculada a média aritmética com os valores do módulo de elasticidade obtidos nas 6 medições. $O$ valor do médio do módulo de elasticidade encontrado foi de $E_{\text {méd }}=2,164 \cdot 10^{10} \mathrm{~Pa}$, ou 21,64 GPa. Mesmo sem o devido tratamento estatístico, este foi o valor adotado para o dimensionamento da viga, uma vez que a proposta da atividade não estava focada no controle dos resultados obtidos devido as circunstâncias de realização do experimento.

O valor médio do módulo de elasticidade encontrado pode ser considerado relativamente elevado para a classe do material escolhido (polímeros). A falta de controle das variáveis do experimento, pode ter contribuído para isso. No entanto, esse fato não exclui a atividade como alternativa metodológica para o ensino do tópico 
da disciplina de Mecânica dos Sólidos, uma vez que o experimento tem fins estritamente didáticos.

Outro fato importante sobre os dados obtidos para o módulo de elasticidade é que estes apresentaram resultados relativamente menores ao passo em que o peso acumulado aumentava. De fato, isso pode estar relacionado principalmente ao fato de a Equação 1 descrever o comportamento do material em regime de pequenas deformações e inclinações.

A Tabela 5 apresenta os parâmetros obtidos a partir dos dados da placa. Vale lembrar que foi adotado o valor da massa específica da madeira por meio de consulta a literatura de $\boldsymbol{\rho}=1320 \mathrm{~kg} / \mathrm{m}^{3}$.

Tabela 5 - Parâmetros obtidos através dos dados referentes à placa

\begin{tabular}{cccc}
\hline $\mathbf{V}\left(\mathbf{m}^{\mathbf{3}}\right)$ & $\boldsymbol{\rho}\left(\mathbf{k g} / \mathbf{m}^{\mathbf{3}}\right)$ & $\mathbf{P p}(\mathbf{N})$ & $\mathbf{T}(\mathbf{N})$ \\
\hline 0,02 & 1320 & 258,72 & 129,36
\end{tabular}

Fonte: Autores (2021)

A partir dos parâmetros da Tabela 5 , foi construído o modelo teórico na ferramenta computacional Ftool. O modelo teórico permitiu o dimensionamento da viga.

Figura 5 - Modelo teórico e reações de apoio

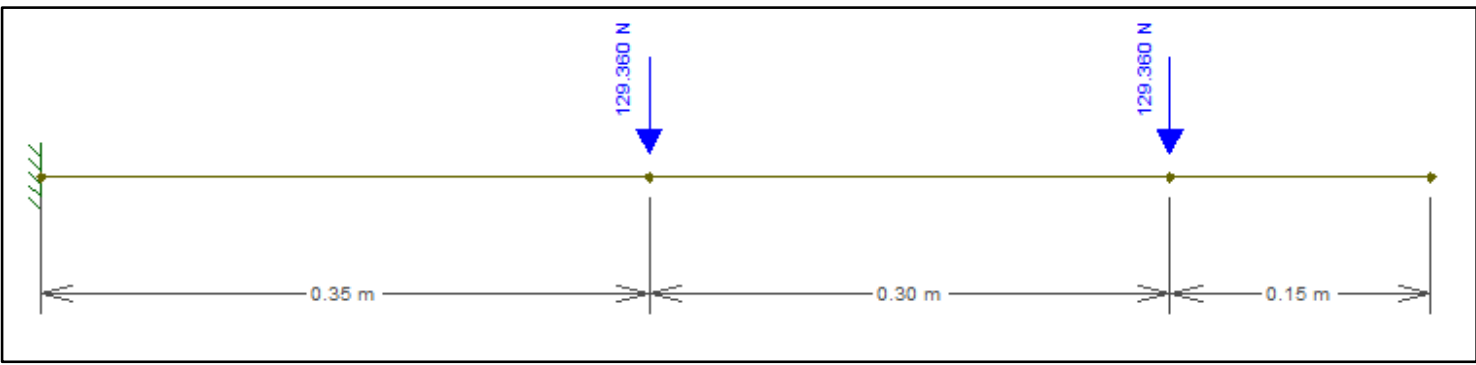

Fonte: Autores (2021)

Para o dimensionamento da viga, foi determinada a deflexão na extremidade livre por conta das duas cargas concentradas em função do diâmetro da viga, $\delta$, a deflexão máxima de projeto permitido por norma, $\delta_{\text {máx }}=\frac{0,8}{250}=0,0032$. Comparando os dois valores, foi encontrado o valor do diâmetro mínimo para atender aos critérios de projeto. A Tabela 6 mostra esses resultados.

Tabela 6 - Deformação total, máxima e diâmetro da viga.

\begin{tabular}{cccc}
\hline $\boldsymbol{\delta}(\mathbf{m})$ & $\boldsymbol{\delta}_{\text {máx }}(\mathbf{m})$ & $\mathbf{d}(\mathbf{m})$ & $\mathbf{d}($ adotado) $(\mathbf{m})$ \\
\hline$\frac{2,0104 \cdot 10^{-8}}{d^{4}}$ & 0,0032 & 0,050 & $\mathbf{0 , 0 6}$ \\
\hline
\end{tabular}

Fonte: Autores (2021)

Após realizar a correlação entre as deformações ao longo da estrutura, e, de acordo com a flecha máxima admitida, obteve-se o valor de $0,050 \mathrm{~m}$ para o diâmetro mínimo da viga. Diante disso, o valor diâmetro adotado foi $0,06 \mathrm{~m}$, com o objetivo de dimensionar a viga com parâmetros que apresentem margem de segurança em relação ao limite de deslocamento imposto. Com o diâmetro adotado, realizou-se novamente a verificação de deslocamento da viga e o resultado pode ser visto na Figura 6. 
Figura 6 - Diagrama de deflexão ao longo da viga

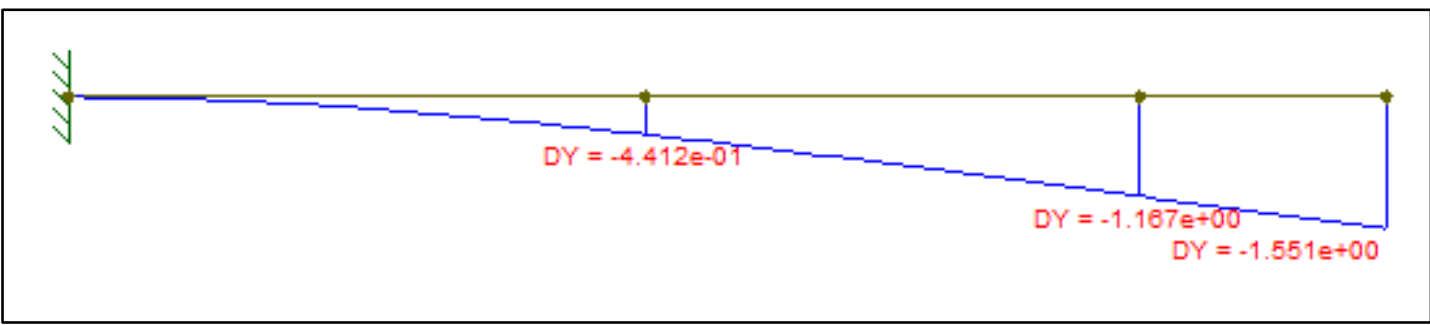

Fonte: Autores (2021)

A partir da análise da Figura 6, é possível observar que o deslocamento máximo na extremidade livre da viga com diâmetro adotado é de $1,551 \mathrm{~mm}$. Como a flecha máxima imposta por norma é de $3,2 \mathrm{~mm}$, a viga atende ao critério do deslocamentolimite.

Vale ressaltar que a atividade propôs o critério de flexa máxima para o dimensionamento da viga, uma vez que para se determinar a tensão de ruptura do material tornaria o experimento mais complexo. Em termos de aulas presenciais, onde o uso do laboratório não esteja registro, seria plausível completar o dimensionamento se utilizando dos demais critérios.

Com a viga dimensionada, foi realizada a análise de tensões. Com o auxílio do FTool, foram determinadas das reações de apoio e plotados os diagramas de esforços internos. Como resultado foram obtidas a reação vertical no ponto de engaste com o valor de 258,72 N e momento fletor atuante de 129,36 N.m. A estrutura em questão não possui esforços normais. O Diagrama de esforço cortante e de momento fleto estão representados na Figura 7.

Com isso, após análise da Figura 7, no caso, dos esforços cortantes e momentos fletores atuantes na estrutura, determinou-se o esforço cortante máximo (Vmáx) e o momento fletor máximo (Mmáx), que correspondem, respectivamente, a $258,72 \mathrm{~N}$ e $129,36 \mathrm{~N} \cdot \mathrm{m}$.

Figura 7 - Diagrama de esforço cortante e diagrama de momento fletor

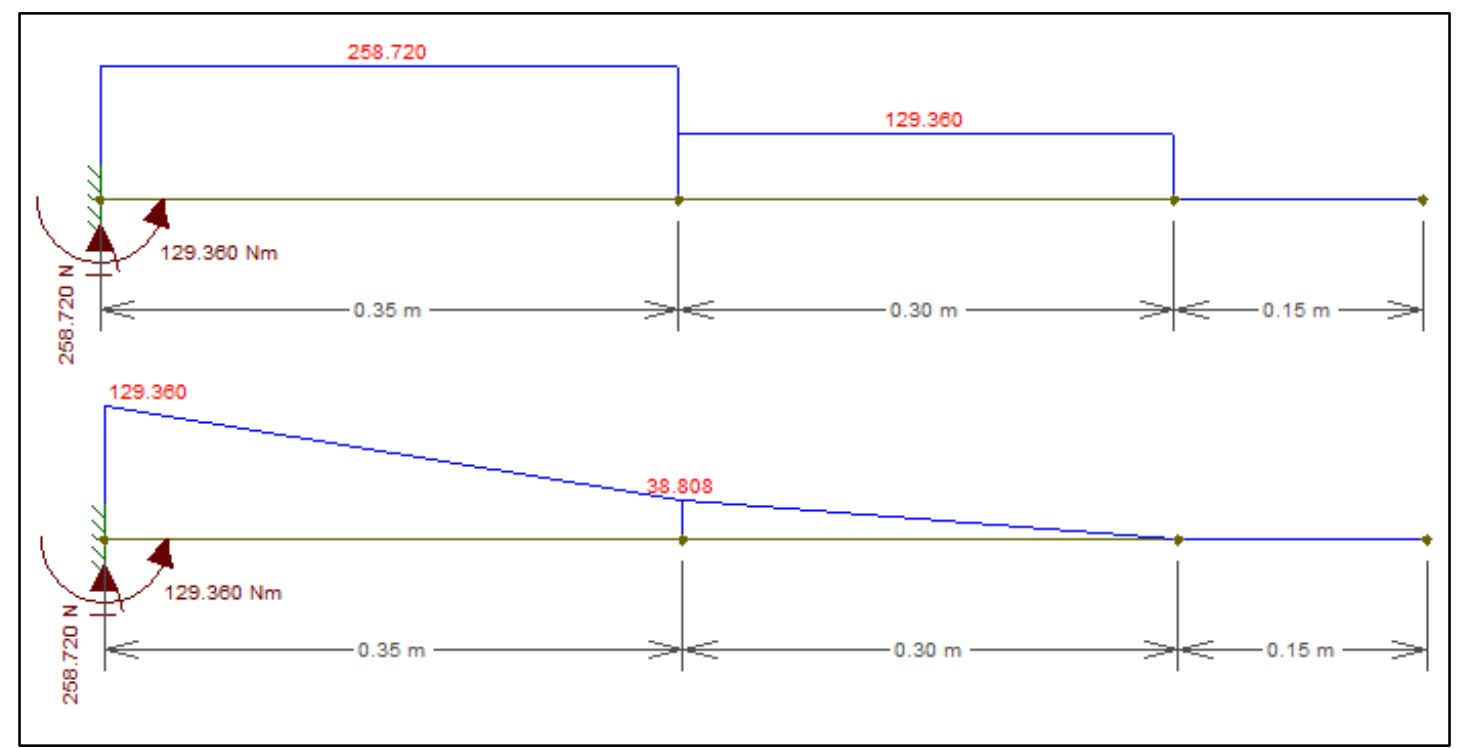

Fonte: Autores (2021) 
Em seguida, foi determinado o módulo da seção (S), o momento de inércia da viga circular considerada no projeto (I), a distância referente à linha neutra (c) e a área da seção transversal (A). Os valores estão apresentados na Tabela 7.

Tabela 7 - Parâmetros dimensionais da viga

\begin{tabular}{cccc}
\hline $\mathbf{S}\left(\mathbf{m}^{\mathbf{3}}\right)$ & $\mathbf{I}\left(\boldsymbol{m}^{\mathbf{4}}\right)$ & $\mathbf{C}(\mathbf{m})$ & $\mathbf{A}\left(\mathbf{m}^{\mathbf{2}}\right)$ \\
\hline $1,38 \cdot 10^{-5}$ & $3,589 \cdot 10^{-7}$ & 0,026 & $2,1237 \cdot 10^{-3}$ \\
\hline Fonte: Autores $(2021)$ & & &
\end{tabular}

Com base nos valores da Figura 7 e da Tabela 7, foram obtidos os valores das tensões máximas normais de compressão, tração e de cisalhamento. A Tabela 8 mostra os resultados obtidos para as tensões máximas.

Tabela 8 - Tensões máximas normais e de cisalhamento

\begin{tabular}{cccc}
\hline$\sigma m a ́ x(\mathrm{~Pa})$ & $\sigma m a ́ x($ compressão) $(\mathrm{Pa})$ & $\sigma m a ́ x(t r a c ̧ a ̃ o)(\mathrm{Pa})$ & tmáx $(\mathrm{Pa})$ \\
\hline $\mathbf{9 , 4 0} \cdot \mathbf{1 0}$ & $-9,40 \cdot 10^{6}$ & $9,40 \cdot 10^{6}$ & $1,62 \cdot 10^{5}$ \\
\hline
\end{tabular}

Fonte: Autores (2021)

Os resultados obtidos permitiram avaliar quais os pontos de tensão máxima da viga. Para a verificação completa das condições da viga, deveria ter sido realizada a comparação com os parâmetros de resistência do material, sejam eles obtidos experimentalmente ou na literatura, porém está análise não fazia parte do escopo da atividade apresentada neste artigo.

Contudo, é importante ressaltar que a proposta da atividade foi atendida satisfatoriamente, uma vez que os estudantes obtiveram êxito na resolução da situação problema proposta e puderam construir o conhecimento de forma prática, aplicando a teoria trabalhada durante as aulas.

\section{CONSIDERAÇÕES FINAIS}

Diante do exposto, a proposta de atividade de Aprendizagem Baseada em Projetos permitiu aos estudantes confrontarem a teoria vista durante as aulas por meio do desenvolvimento de projeto da viga, simulando de forma didática uma situação prática. Assim, foi possível a determinação experimental do módulo de elasticidade de material não convencional, possibilitando o dimensionamento da viga por meio do critério normativo do deslocamento-limite.

Além disso, a realização da atividade possibilitou a verificação, de maneira prática, dos valores máximos de tensões normais e de cisalhamento atuantes da viga. Ainda, a aplicação da atividade foi de suma para importância para a utilização de ferramentas computacionais que potencializam a análise e as etapas de estudo.

Por fim, o artigo exerce papel fundamental para a produção de conhecimento acerca da educação em engenharia civil, uma vez que fortalece as discussões sobre as metodologias e estratégias pedagógicas utilizadas no processo de ensino aprendizagem.

\section{REFERÊNCIAS}

ASSOCIAÇÃO BRASILEIRA DE NORMAS TÉCNICAS. NBR 15.575: Edificações habitacionais - Desempenho. Rio de Janeiro, 2013. 
BEER, F. P. et al. Mecânica dos materiais. 5. ed. Editora McGraw-Hill, 2011.

CHISTOFORO, A. L; RIBEIRO FILHO, S. L. M. et al. Metodologia para o cálculo dos módulos de elasticidade longitudinal e transversal em vigas de madeira de dimensões estruturais. Rede de Revistas Científicas da América Latina, Caribe, Espanha e Portugal. Santa Maria, V. 43, n. 4, p. 610 - 615, 2013.

CIPOLLA, L. E. Aprendizagem baseada em projetos: a educação diferenciada para o século XXI. 17v. n(3). Científica. Rio de Janeiro, 2016.

DAINEKO, Y.; DMITRIYEV, V.; IPALAKOVA, M. Using virtual laboratories in teaching natural sciences: an example of physics courses in university. Computer Applications in Engineering Education, 2017.

FEITOSA, A. M. Realidade aumentada no ensino de física. 165 f. Dissertação (Mestrado) - Universidade Federal de Alagoas - UFAL, Maceió, 2020.

HIBBELER, R. C. Resistência dos materiais. 7. ed. Editora Pearson, 2010.

IBÁÑEZ, M.B.; DELGADO-KLOOS, C. Augmented reality for STEM learning: a systematic review. Computers \& Education, 2018.

KRÜGER, M.; DA SILVA, G. Projeto estrutural de uma residência de dois pavimentos. Ignis: Periódico Científico de Arquitetura e Urbanismo, Engenharias e Tecnologia da Informação, p. 21-37, 2019.

TIMOSHENKO, S. P.; GERE, J. E. Mecânica dos sólidos. 3. ed. v. 1. Rio de Janeiro: Editora Livros Técnicos e Científicos, 1983.

\section{ACTIVITY OF PROJECT BASED LEARNING IN CIVIL ENGINEERING: PROPOSAL FOR EXPERIMENTAL ANALYSIS OF YOUNG'S MODULUS AND BEAM DESIGN}

Abstract: This paper aims to present the results obtained in the activity applied in the Solid Mechanics III class of the Civil Engineering undergraduate program at the Federal Institute of Alagoas - IFAL, using concepts of Project Based Learning - PBL as a methodological alternative during emergency remote education. In general, the methodological procedures of the PBL Activity can be divided into four stages: delimitation of the problem situation, didactic prototype of an experiment for the Young's modulus, beam design and stress analysis. Initially, the students received the proposal for the activity that consists of designing the support beam for a plate from the material of a chosen object, in this case, a common polymeric ruler. In addition, the students adopted the dimensions and material of the plate. From the chosen ruler, the students built the didactic prototype to perform an experiment to determine the Young's modulus of the material, using the concepts of solid mechanics worked during the classes. Based on the average value of the Young's modulus of the material and the data adopted, it was possible to design the plate support beam and then elaborate the beam design. From the design parameters, stress analysis was carried out on the beam. In general, the results of the activity, as a methodological proposal, can be 
considered satisfactory, as the students were able to solve the proposed problem situation, elaborate the beam design and analyze the beam, relating theory and practice.

Keywords: Project based learning. Solid Mechanics. Beam. 